\title{
PROBABILISTIC TAGGING WITH FEATURE STRUCTURES
}

\author{
André Kempe \\ University of Stultgart, Institute for Computational Linguistics, \\ Azenbergstraße 12, 70174 Stuttgart, Germany, kempe@ims.uni-stuttgart.de
}

\begin{abstract}
The described tagger is based on a hidden Markov model and uses tags composed of features such as partof-speech, gender, etc. The contextual probability of a tag (statce transition probability) is decluced from the contextual probabilities of its feature-value-pairs.

This approach is advantageons when the available training corpus is small and the tag set large, which can be the case with morphologically rich languages.
\end{abstract}

\section{INTRODUCTION}

The present article describes at probabilistic tagger based on a hidden Markov model (IIMM) (Rabiner, 1990) and employs tags which are fenture structures. 'llicir features concern part-of-spech (POS), gender, number, etc. and have only atomic values.

Usually, the contextual probability of a tag (state transition probability) is estimated dividing a trigram frequency by a bigram frequency (second order MMM). With a large tag set resulting from the fact that the tags contain besides of the POS it lot of morphological information, and with only a small training corpus available, most of these frequencies are too low for an exact estimation of contextual probabilities.

Our feature structure tagger estimates these probabilities by connecting contextual probabilities of the single feature-value-pairs (fv-pairs) of the Lags (cf. sec. 2).

Starting point for the implementation of the feature structure tagger was a second-order-IIMM tagger (trigrams) based on a modified version of the Viterli algorithm (Viterbi, 1967; Church, 1988) which we had earlier implemented in C (Kempe,1994). There we modified the calculus of the contextual probabilities of the tags in the above-described way (cf sec. 1).

$A$ test of both taggers under the same conditions on a French corpus ${ }^{1}$ has shown that the feature struchure tagger is clearly better when the available training corpus is small and the tag set is large but the lags are decomposable into relatively few fv-pairs. The latter can be the case with morphologically rich languages when the tags contain a lot of morphological information (cf. sec. 5).

\footnotetext{
${ }^{1}$ I an much obliged to Achim Stein and Leo Wanner, Romance Dept., Univ. Stuttgart, Germany, for provicling the corpus and a dictionary.
}

\section{MATHEMATTCAL BACK- GROUND}

In order to assign tags to a word sequence, a IIMM can be used where the tagger selects among all possible tag sequences the most probable one (Garside, Leech and Sampson, 1987; Church, 1988; Brown et al., 1989; Rabiner, 1990). The joint probability of a tag sequence $\bar{t}=t_{0} \ldots t_{N-1}$ given a word sequence $\bar{w}=w_{0} \ldots w_{N-1}$ is in the case of a second order IIMM:

$$
\begin{aligned}
& p(\bar{l}, \bar{w})=\pi_{t_{0}} t_{1} \cdot p\left(w_{0} \mid t_{0}\right) \cdot p\left(w_{1} \mid t_{1}\right) \cdot \\
& \prod_{i=2}^{N-1}\left(p\left(w_{i} \mid t_{i}\right) \cdot p\left(t_{i} \mid t_{i-2} t_{i-1}\right)\right)
\end{aligned}
$$

'The term $\pi_{t_{0} t_{8}}$ stands for the initial state probability, i.e. the probability that the sequence begins with the first two tags. $N$ is the number of words in the sequence, i.e. the corpus size. The term $p\left(w_{i} \mid l_{i}\right)$ is the probability of a word $w_{i}$ in the context of the assigned $\operatorname{tag} t_{i}$. It is called observation symbol probability (lexical probability) and can be estimated by:

$$
p\left(u_{i} \mid t_{i}\right)=\frac{\int\left(w_{i} t_{i}\right)}{f\left(t_{i}\right)}
$$

The second order state transition probability (contextual probability) $p\left(t_{i} \mid t_{i-2} t_{i-1}\right)$ in formula (1) expresses how probable it is that the tar $t_{\text {; }}$ appears in the context of its two preceding tags $t_{i-2}$ and $t_{i-1}$. It is usually estimated as the ratio of the frequency of the trigram $\left\langle l_{i-2}, l_{i-1}, t_{i}\right\rangle$ in a given training corpus to the lieguency of the higram $\left\langle t_{i-2}, t_{i-1}\right\rangle$ in the same corpus:

$$
p\left(t_{i} \mid l_{i-2} l_{i-1}\right)=\frac{f\left(l_{i-2} l_{i-1} l_{i}\right)}{\int\left(l_{i-2} l_{i-1}\right)}
$$

With a large tag set and a relatively small handtagged training corpus formula (3) has an importint disadvantage: 'Tho majority of transition probabilities cannot be estimated exactly because most of the possible trigrams (sequences of three consecutive tags) will not appear at all or only a few times".

In our example we have a lirench training corpus of 10,000 words tagged with a set of 380 different targs which could form $386^{3}=57,512,456$ different trigrams, but because of the corpus size no more than 10,000-2 trigrauns can appear. Actually, their number was only 4,815 , i.e. $0.008 \%$ of all possible

\footnotetext{
${ }^{2} \Lambda$ detialed description of prohlems caused by snall and zero fiecuencies was given by Gale and Church (1989)
} 
ones, because some of them appeared more than once (table 1).

\begin{tabular}{|l|lc|}
\hline $\begin{array}{l}\text { frequency } \\
\text { range }\end{array}$ & $\begin{array}{l}\text { number and percentage } \\
\text { of trigrams in the range }\end{array}$ \\
\hline \hline 128 & 1 & $(0.021 \%)$ \\
\hline $64-127$ & 2 & $(0.042 \%)$ \\
\hline $32-63$ & 13 & $(0.26 \%)$ \\
\hline $16-31$ & 43 & $(0.89 \%)$ \\
\hline $8-15$ & 119 & $(2.5 \%)$ \\
\hline $4-7$ & 282 & $(5.9 \%)$ \\
\hline $2-3$ & 860 & $(18 \%)$ \\
\hline 1 & 3,495 & $(73 \%)$ \\
\hline \hline sum & 4,815 & $(100 \%)$ \\
\hline
\end{tabular}

Table 1: Thigram count from a French training corpus of 10,000 words

When we divide e.g. a trigram frequency 1 by a bigram frequency 2 according to formula (3) we get the probability $p=0.5$ but we cannot trust it to be exact because the frequencies it is based on are too small.

We can take advantage of the fact that the 386 tags are constituted by only 57 different fv-pairs concerning POS, gender, number, etc. If we consider probabilistic relations between single fv-pairs then we get higher frequencies (fig. 2) and the resulting probabilities are more exact.

From the equations

$$
\left\{t_{i}\right\}=\left\{e_{i 0} \cap e_{i 1} \ldots \cap e_{i, n-1}\right\}=\left\{\bigcap_{k=0}^{n-1} e_{i k}\right\}
$$

where $t_{i}$ means a tag and the $e_{i k}$ symbolize its $[v$-pairs and

$$
\begin{array}{r}
p\left(\bigcap_{k=0}^{n-1} e_{i k} \mid C_{i}\right) \cdot p\left(C_{i}\right)=p\left(\left(\bigcap_{k=0}^{n-1} e_{i k}\right) \cap C_{i}\right) \\
\left(C_{i}\right) \cdot p\left(e_{i 0} \mid C_{i}\right) \cdot p\left(e_{i 1} \mid C_{i} \cap e_{i 0}\right) \cdot \\
\cdots \cdot p\left(e_{i, n-1} \mid C_{i} \cap \bigcap_{k=0}^{n-2} e_{i k}\right)
\end{array}
$$

whet $C_{i}$ means the context of $t_{i}$ and contains the tags $t_{i-2}$ and $t_{i-1}$ follows

$$
p\left(t_{i} \mid C_{i}\right)=p\left(e_{i 0} \mid C_{i}\right) \cdot \prod_{i=1}^{n-1} p\left(e_{i k} \mid C_{i} \cap \bigcap_{j=0}^{k-1} e_{i j}\right)
$$

The latter formula $\mathrm{a}^{3}$ describes the relation between the contextual probability of a tag and the contextual probabilities of its fv-pairs.

The unification of morphological features inside a noun phrase is accomplished indirectly. In a given context of fv-pairs the correct fv-pair obtains the probability $p=1$ and therefore will not infuence the probability of the tag to which it belongs (e.g. $p_{1}^{*}($ onum:SG $\mid \ldots)=1$ in fig. 2). $A$ wrong fv-pair would obtain $p=0$ and make the whole tag impossible.

\footnotetext{
${ }^{3}$ suggested by Mats Rooth, IMS, Univ.Stuttgart, Germany
}

\section{TRAINING ALGORITHM}

In the training process we are not interested in analysing and storing the contextual probabilities (state transition probabilities) of whole tags but of single fv-pairs. We note them in terms of probabilistic Sealure relations (PFR):

$$
\operatorname{PFR}:\left\langle e_{i} \mid C_{i}^{s u b} ; p\left(e_{i} \mid C_{i}^{s u b}\right)\right\rangle
$$

which later, in the tagging process, will be combined in order to obtain the contextual tag probabilities.

The term $e_{i}$ in formula $(7)$ is a fv-pair. $C_{i}^{u t}$ is a reduced context which contains only a subset of the fv-pairs of a really appearing context $C_{i}$ (fig. 1). $C_{i}^{s u b}$ is obtained from $C_{i}$ by eliminating all fv-pairs which do not influence the relative frequency of $e_{i}$, according to the condition:

$$
p\left(e_{i} \mid C_{i}^{s u b}\right) / p\left(e_{i} \mid C_{i}\right) \in[1-\epsilon, 1+\epsilon]
$$

The considered fv-pair has nearly ${ }^{4}$ the same probability in the complete and in the reduced contexts, i.e. $C_{i}$ does not supply more information about the probability of $e_{i}$ than $C_{i}^{s u b}$ does.
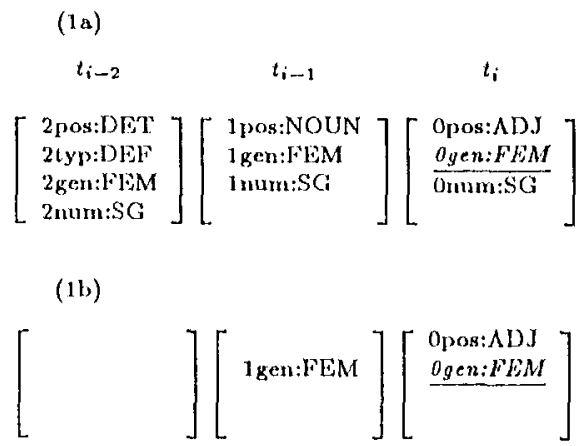

Figure 1: (a) Complete context $C_{i}$ and (b) reduced context $C_{i}^{* u b}$ of the feature-value-pair $e_{i}=0$ ogn:FEM

In the example (fig. 1a) we consider the fv-pair Ogen:Fl'M. Within the given training corpus, its probability in the complete context $C_{i}$, i.e. in the context of all the other fv-pairs of figure $1 \mathrm{a}$, is $\gamma_{0}^{*}=14 / 41=1$ (c. $p_{0}^{*}$ in lig. 2).

The presence of 1 num:SG in tag $t_{i-1}$ does not infuence the probability of ogen:FEM in tag $t_{i}$. Therefore Inum:SG can be eliminated. Only fv-pairs which really have an influence remain in the context. The reduced context $C_{i}^{u b b}$ with less fv-pairs, which we obtain this way, is more general (fig. 1b).

In the given training corpus, the probability of Ogen:FEM in the context $C_{i}^{s u b}$ is $p_{0}=170 / 174=0.997$ (cf. $p_{0}$ in $P F R_{0}$ in fig. 2), which is near to $p_{0}^{*}=1$. 'The reduced context $C_{i}^{s i b}$ is used to form a PFR which will be stored.

\footnotetext{
${ }^{4}$ A small change in the probability caused by the elimination of fv-pairs from the context is admitted if it does not exceed a defined small percentage $c$. (We used $\epsilon=3 \%$.)
} 
We sec in the use of reduced contexts instead of complete ones two advantages:

(1) $\Lambda$ great number of complete contexts containing many fv-pairs can lead after elimination of irrelevant fv-pairs to the same PFR, which makes the number of all possible PFls much smaller than the number of all possible trigrams (cf, sec. 2).

(2) The probability of a fv-pair can be estimated more exactly in a reduced context than in a complete one because of the higher frequencies in the first case.

\section{The Generation of PFls:}

In the training process we first extract from a training corpus a set of trigrams where the tags are split, up into their fv-pairs. From these trigrams a set of PPRs is generated separately for every fv-pair $e_{i}$. We examined four different methods for this procedure:

Method 1-3: For every trigram we generate all possible subsets of its fv-pairs. Many trigrams, e.g. if they differ in only one fv-pair, have most of their subsets of fv-pairs in common. Both the complete trigrams and the subsets, constitute together the set of contexts and subcontexts $\left(C_{i}\right.$ and $\left.C_{i}^{\text {sub }}\right)$ wherein a fv-pair could appear. To generate PFlis for a given [v-pair, we preselect and mark those (sub-)contexts which are supposed to have an influence on the contextual probability of the (v-pair. $A$ (sub-) context will not be preselected if its frequency is smaller than a defined threshold. We use diflerent ways for the preselection:

Melhod 1: $\Lambda$ (sub-)context will be preselected if the considered fv-pair itself or an fv-pair betonging to the same feature type ever appears in this (sub-)context. E.g., if gen:MAS appears in a certain (sub-)context then this (sub-)context will be preselected for gen:Fl'M too. Furthemore, it is possible to impose special conditions on the preselection, e.g. that is (sub-) context can only be preselected if it contains a POS feature in tar $l_{i}$ and $l_{i-1}$ (cf. lig. la: Opos and $I$ pos).

Methool 2: In order to preselect (sub-) contexts for an fv-pair, we generate a decision trec" (Quinlan, 1983) where the feature of the fv-pair, e.g. gen, num ete., serves to classify all existing (sub-) contexts. Legr, num produces three classes of contexts: those containing the fv-pair onum:SG, those with Onum:PI and those without a Onum feature. We assign to the tree nodes other features than this upon which the classification is based. The root node is labeled with the feature from which we expect most information about the probability of the currently considered feature. 'The values of the root node feature are assigned to the branchess starting at the root node. We continue the branching until there remain no features with an expected information gain and a frequency higher than defined

\footnotetext{
${ }^{5}$ suggested by Jelmut Schmid, IMS, Univ. Stultgart, Cermany. For reasons of space we explain only how we employ decision trees for our purposes. For details about the antomatic: generation of such trees see Quinlan (1983).
}

thresholds. 'To every leaf of the tree corresponds at (sub-)context which will bo marked and thus preselected for further analysis.

Method 3: For each fv-pair concerning POS we preselect every (sub-) context containing only POS lea tures in tag $t_{i-2}$ and $t_{i-1}$ (classical POS trigram), e.g. 2pos:PREP tpos:DET for opos:NOUN. For the other fv-pairs wo mark every (sub-) context containing any fv-pair of the same type in the previous tag $t_{i-1}$ and any POS features in tag $t_{i-1}$ and $t_{i}$, e.g. 1pos:DE' Igen:FeM Opos: NOUN for Ogen:FWM.

With the methods $1-3$, we next eliminate from every preselected (sub-)context all fv-pairs which in the above described sense do not influence the relative frequency of the currently considered fv-pair (eq. 8).

Method 4: From the set of trigrams extracted from a training corpus we generate separately for every fvpair, a binary-branched decision tree which slatl describe various contextual probabilities of this [v-pair. The tree is gencrated on a modified version of the ID3 algorithm (Quinlam, 1983) and is similar to the one described by Schmid (1994).

We slart with a binary classification of all trigrams based on the considered lv-pair. Leg., a classification for gen: $F^{\prime} E M$ will divide the set of trigrans in two subsets, one where the trigrams contain $0 y c n: F F M$ in the tag $l_{i}$ and one where they do not.

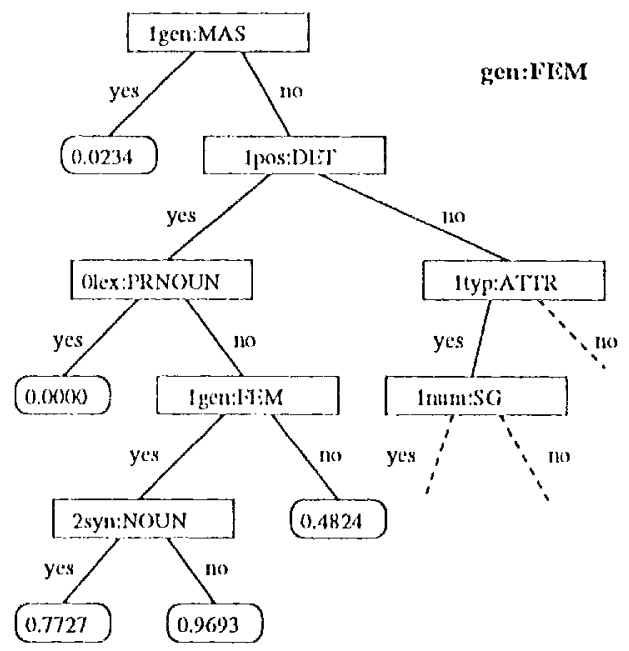

Figure 3: Decision tree for the fv-pair Ogen:Flim (livery number is a probability of Ogen:FiM in the context described by the path from the root node to the node labeled with the number.)

The tree is built up recursively (fig. 3). At each step, i.e. with the construction of each node, we test which one of the other fv-pairs delivers most information concerning the above-described classification. 'The current node will be labeled with this [v pair. One of its two branches concerns the trigrans which con- 


$$
\begin{aligned}
& p \text { (Ogen:FEM 0num:SG 0pos:ADJ | 1 gen:F'FM 1num:SG 1pos:NOUN } \\
& \text { 2gen:FEM 2num:SG 2pos:DET 2typ:DEF) }=14 / 298=\underline{0.148} \\
& p_{0}^{*}(\text { gentFEM | } 0 \text { num:SG 0pos:ADJ 1 gen:FEM } 1 \mathrm{mum}: S G \text { 1 pos:NOUN } \\
& \text { 2gen:FEM 2num:SG 2pos:DET 2typ:DEF) }=14 / 44=1.0 \\
& \text { PFRo : (Ogen:FEM | Opos:ADJ 1gen:FEM ; } p_{0}=170 / 174=0.977 \text { ) } \\
& p_{1}^{*} \text { ( Onum:SG | 0gen:FEM 0pos:AD) J 1 gen:FlEM } 1 \text { num:SG 1 pos:NOUN } \\
& 2 \text { gen:FEM 2num:SG 2pos:DE: 2typ:DEF) }=11 / 41=1.0 \\
& \text { PFR } \left._{1}: \text { (0mum:SG |0pos:ADJ 1num:SG 2pos:DET ; } p_{1}=96 / 96=1.0\right\rangle \\
& p_{2}^{*}(0 \text { pos:ADJ 1 1gen:FEM } 1 \text { num:SG 1pos:NOUN } \\
& \text { 2gen:FEM 2num:SG 2pos:DET 2typ:DEF) }=44 / 298=0.148 \\
& \mathrm{PFR}_{2}: \text { (0pos:ADJ | 1gen:FEM 1pos:NOUN 2pos:DET; } p_{2}=69 / 465=0.148 \text { ) } \\
& \prod_{i=0}^{2} p_{i}=\underline{0.145}
\end{aligned}
$$

The position index at the beginning of every feature-value-pair indicates the tag to which it belongs; e.g. Ogen:FEM belongs to tag $t_{i}$ and $2 n u m: S G$ to $t_{i-2}$.

Figure 2: Decomposition and reconstruction of a contextual tag probability (state transition probability) using probabilistic fcalure relations (PFR.)

tain the fv-pair, the other branch concerns the trigrams which do not contain it. The recursive expansion of the tree stops if either the information gained by consulting further fv-pairs or the frequencies upon which the calculus is based are smaller than defined thresholds.

\section{TAGGING ALGORITHM}

Starting point for the implementation of a feature structure tagger was a second-order-IIMM tagger (trigrams) based on a modified version of the Viterbi algorithm (Viterbi, 1967; Church, 1988) which we had earlier implemented in C (Kempe ,1994). There we replaced the function which estimated the contextual probability of a tag (state transition probability) by dividing a trigram frequency by a bigram frequency (eq. 3) with a function which accomplished this calculus either using PFRs in the above-described way (eq.s 6,7 ) or by consulting a decision tree (fig. 3).

To estimate the contexlual probability of a lag we have to know the contextual probabilities of its $\mathrm{fv}$ pairs in order to multiply them (eq. 6).

Using PFRs generated by method 1 or 2 , when e.g looking for the probability $p_{2}^{*}$ (0pos:ADJ f...) from figure 2, we may find in the list of PPRs, instead of a PFR which would directly correspond (but is not stored), the two PFRs

$$
\begin{aligned}
& \text { 〈0pos:ADJ | 1gen:FEM 1pos:NOUN 2pos:DFI; } \\
& \left.p_{1}=0.148\right\rangle \\
& \text { 〈0pos:ADJ | 0num:SG 1num:SG 1syn:NOUN 2syn:DET; } \\
& \left.p_{2}=0.411\right\rangle
\end{aligned}
$$

Both of them contain subsets of the fv-pairs of the required complete context and could therefore both be applied. In such case we need to know how to combine $p_{1}$ and $p_{2}$ in order to get $p\left(=p_{2}^{*}\right.$ in fig. 2$)$.

$\Lambda$ s there exists no mathematical relation between these three probabilities, we simply average $p_{1}$ and $p_{2}$ to get $p$ because this gives as good tagging results as at number of other more complicated approaches which we examined.

PFRs generated by method $\mathbf{3}$ do not create this problem. For every complete context only one PFR is stored.

When we use the set of decision trees generated by method 4, we obtain for every fv-pair in every possible context only one probability by going down on the relevant branches until a probability information is reached.

In opposition to the PFRs of the other methods, the decision trees also contain negative information about the context of an fv-pair, i.e. not only which [v-pairs have to be in the context but also which ones must be absent.

\section{TAGGING RESULTS}

In the training and tagging process we experimented with different values for parameters like: minimal admitted frequency for presclection, admitted percentual difference $\epsilon$ between probabilities considered to be equal, etc. (cf. sec. 3).

The feature structure tagger was trained on the French 10,000 words corpus already mentioned in table 1, with the four different training methods (sec. 3). When tagging a 6,000 words corpus ${ }^{6}$ with an average ambiguity of 2.63 tags per word (after the dietionary

\footnotetext{
${ }^{6}$ No overlap between trining and test corpora.
} 
look-up) we obtained in the best case an accuracy of $88.89 \%$ (table 2).

\begin{tabular}{|c|c|c|c|c|c|c|}
\hline \multirow{2}{*}{$\begin{array}{l}\text { tag- } \\
g c r\end{array}$} & \multicolumn{2}{|c|}{ training corpus } & \multicolumn{2}{|c|}{ tag set } & \multirow{2}{*}{$\begin{array}{l}\text { IIMM } \\
\text { order }\end{array}$} & \multirow{2}{*}{$\begin{array}{l}\text { tabging } \\
\text { accuracy }\end{array}$} \\
\hline & $\begin{array}{l}\text { number } \\
\text { of words }\end{array}$ & $\begin{array}{l}\text { lan- } \\
\text { guag } \\
\end{array}$ & tags & $\begin{array}{l}\text { fv- } \\
\text { prs. }\end{array}$ & & \\
\hline $\mathrm{T}$ & $, 000,000$ & En & 47 & - & 1 & \\
\hline$\Gamma$ & & & 47 & - & & $\%$ \\
\hline $\mathrm{T}$ & 10,000 & French & 386 & 57 & 1 & $\%$ \\
\hline 1 & 001 & h & $386^{\circ}$ & 57 & & $3 \%$ \\
\hline $\mathrm{PT}$ & & Fre & 386 & 57 & 2 & $3.81 \%$ \\
\hline fsT1 & & Fre & 386 & 57 & & $8.53 \%$ \\
\hline $\mathrm{sT2}$ & & rench & 386 & 57 & $=$ & $88.89 \%$ \\
\hline 113 & & & 386 & 57 & 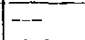 & $88.41 \%$ \\
\hline $\bar{s} 14$ & 10,000 & Frencli & 386 & 57 & $1-$ & $88.14 \%$ \\
\hline
\end{tabular}

$t^{\prime} T \rightarrow$ "traditional" IIMM-tagger,

lp $T \rightarrow$ "Tagger" considering only lexical probabilities,

$f s T 1 . .1 \rightarrow$ feature structure tagger

trained with method $1 . .4$,

IIMM order $1 \rightarrow$ bigrams, $2 \rightarrow$ trigrams

Table 2: Comparison of the tagging accuracy with different taggers, corpora, tag sets and IIMM orders

Comparatively, we used a "traditional" IIMMtagger (cf. sec. 4) on the same training and test corpora and got an accuracy of $83.23 \% 7$, i.e. the error rate was about $50 \%$ higher than with the feature structure tagger (table 2).

When we used a tool which always selects the lexically most probable tag without considering the context we obtained an accuracy of $83.81 \%$, which is even better than with the "traditional" IIMM-tagger.

Provided with enough training data and working on a small tag set, our "traditional" tagger got an accuracy of $96.16 \%$ (Kempe ,1994), which is usual in this case (Cutting et al.,1992). The linglish test corpus we used here had an average ambignity of 2.61 tags per word which is amazingly similar to the ambiguity of the French corpus.

The fealure structure tagger is clearly bether when the available training corpus is small and the targ set large but the tags are decomposable into few fv-pairs.

\section{FURTHER RESEARCH}

We intend to search for other similar models while keeping in mind the basic idea described above: Splitting up a tag into fv-pairs and deducing its contextual probability from the contextual probabilities of its fvpairs.

Furthermore, it may be preferable to split up the tags only when the frequencies are too small ${ }^{8}$.

\footnotetext{
${ }^{7}$ For a similar experinent for German $(20,000$ words l raining corpus, 689 tags, trigrams) an accuracy of $72.5 \%$ has been reported (Wothke et al., 1993, p. 21).

${ }^{8}$ suggested by Ted Briscoe, Rank Xerox Research Centre, Grenoble, France
}

\section{References}

Brown, P.F. et al. (1989). A Statistical Approach to Machine Translation. Technical Report, RC 14773 (\#66226) 7/17/89, IBM Research Division.

Church, K.W. (1988). A Stochastic Parts Program and Noun Phrase Parser for Unrestricted Text. In Proc. 2nd Conference on Applied Nalural Language I'rocessing, ACL., pp. 136-143.

Cutting, D. et al. (1992). A Practical Part-of-Speecl 'Tagger. In Proc. $3 r d$ Conference on Applied Nalural Language Processing, $A C I$. Trento, Thaly.

Gale, W.A. and Church, K.W. (1989). What's Wrong with Adding One?. Statistical Research Reports, No. 90, AT\&'I Bell Jaboratories, Murray IIill.

Garside, R.., Jecel, G. and Sampson, G. (1987). 'The: Computational Analysis of English: $\Lambda$ Corpus-based Approach. Louklon: Longman.

Kempe, A. (1994). A Probabilistic Tagger and an Analysis of 'Tagging Errors. Research Report. IMS, Univ. of Stuttgart.

Quinlan, J.R. (1983). Learning Eflicient Classification Procedures and 'lheir $A$ pplication to Chess Frud Games. In Michalski, R., Carbonell, J. and Mitchell 'l. (lds.) Machine Lecrning: An artificial intelligence approach, pp. 463-482. San Mateo, Calliformia: Morgan Kaufmann.

Rabiner, L.R. (1990). A Tutorial on Hidden Markov Models and Selected $\Lambda$ pplications in Speech Recognition. In Waibel, $\Lambda$ and Lec, K.F. (Wds.) Readings in Speech Recognilion. San Mateo, California: Morgan Kaufmann.

Schmid, II. (1994). Probabilistic Part-of-Speach 'Tagring Using Decision 'Trees. Research Report. IMS, Univ. of Stuttgart.

Viterbi, A.J. (1967). Wrror Bounds for Convolutional Codes and an $\Lambda$ symplotical Optimal Decoding Algorithrn. In P'roccadings of IELE, vol. 61, pp. 268-278.

Wothke, K. et al. (1993). Statistically Based Automatic 'laggring of German Text, Corpora with Partsof-Speech - Some Experiments. Rescarch Report, 1)oc. No. TR 75.93.02. Ileidelberg Scientific Center, IBM Germany. 